\title{
DANTi: Detect and Avoid iN The Cockpit
}

\author{
James Chamberlain ${ }^{1}$ \\ Sunrise Aviation, Inc., Newport News, VA, 23601-1359 \\ María Consiglio² and César Muñoz ${ }^{3}$ \\ NASA Langley Research Center, Hampton, VA, 23681-2199
}

\begin{abstract}
Mid-air collision risk continues to be a concern for manned aircraft operations, especially near busy non-towered airports. The use of Detect and Avoid (DAA) technologies and draft standards developed for unmanned aircraft systems (UAS), either alone or in combination with other collision avoidance technologies, may be useful in mitigating this collision risk for manned aircraft. This paper describes a NASA research effort known as DANTi (DAA iN The Cockpit), including the initial development of the concept of use, a software prototype, and results from initial flight tests conducted with this prototype. The prototype used a single Automatic Dependent Surveillance - Broadcast (ADS-B) traffic sensor and the own aircraft's position, track, heading and air data information, along with NASA-developed DAA software to display traffic alerts and maneuver guidance to manned aircraft pilots on a portable tablet device. Initial flight tests with the prototype showed a successful DANTi proof-of-concept, but also demonstrated that the traffic separation parameter set specified in the RTCA SC-228 Phase I DAA MOPS may generate excessive false alerts during traffic pattern operations. Several parameter sets with smaller separation values were also tested in flight, one of which yielded more timely alerts for the maneuvers tested. Results from this study may further inform future DANTi efforts as well as Phase II DAA MOPS development.
\end{abstract}

\section{Introduction}

$\mathrm{P}$ ILOTS and airborne passengers have been concerned about mid-air collisions (MACs) and near mid-air collisions (NMACs) between aircraft arguably since multiple aircraft began sharing the same airspace more than a century ago. The concern is heightened near convergence points such as airports and, while better air traffic control (ATC) services and traffic awareness/collision avoidance systems ${ }^{1-6}$ have improved the NMAC/MAC safety record over time, a non-trivial safety hazard still persists. The National Transportation Safety Board (NTSB) records contain 112 MAC reports (excluding balloon accidents) in the United States between January 2000 and June 2010, and over the same time period Aviation Safety Information Analysis and Sharing (ASIAS) data contain 3586 NMAC reports (2059 from the ASIAS Aviation Safety Reporting System (ASRS) database and 1527 from the ASIAS NMACs database) ${ }^{7}$. While some of these NMAC reports may be redundant and there may be some over-reporting for various reasons ${ }^{7}$, the MAC and NMAC totals are still significant. The same analysis ${ }^{7}$ shows that the airport environment is where most MACs occurred (59\% of NTSB reports) and most NMACs were reported (64\% of ASRS reports and $47 \%$ of NMACs database reports). A Federal Aviation Administration (FAA) data analysis ${ }^{8}$ states that the majority of in-flight collisions occur within five miles of an airport with $77 \%$ occurring below 1000 feet ( $49 \%$ below 500 feet), "imply[ing] accurately that in-flight collisions generally occur in the traffic pattern and primarily on final approach.”

The primary NMAC/MAC mitigation in visual flight conditions is a pilot's regulatory responsibility to see and avoid other aircraft and to remain "well clear" of them. ${ }^{9}$ This see-and-avoid responsibility may be the only mitigation in some relatively high collision risk flight situations such as arriving to or departing from busy non-towered airports where ATC services are unavailable. An active onboard collision avoidance system such as a Traffic Alert and Collision Avoidance System (TCAS II), ${ }^{3,4}$ if available, is also of limited use in such situations since its Resolution Advisory (RA) guidance for collision avoidance is inhibited below 1000 feet above ground level and, in any case, TCAS II will only issue collision avoidance guidance against transponder-equipped intruder aircraft. Traffic advisory

\footnotetext{
${ }^{1}$ President and Aerospace Research Engineer, 225 Rockingham Drive, AIAA Member.

${ }^{2}$ Research Computer Scientist, Safety Critical Avionics Systems Branch, Mail Stop 130, AIAA Member.

${ }^{3}$ Research Computer Scientist, Safety Critical Avionics Systems Branch, Mail Stop 130, AIAA Senior Member.
}

American Institute of Aeronautics and Astronautics 
systems based on Automatic Dependent Surveillance - Broadcast (ADS-B) have been introduced recently ${ }^{5,6,10}$ such as the ADS-B Traffic Advisory System (ATAS, formerly known as Traffic Situation Awareness with Alerts, or TSAA) that may provide useful mitigation against ADS-B Out equipped intruders, although these systems provide only traffic awareness and do not provide guidance to remain well clear of these intruders. The MAC/NMAC data indicate that pilots' see-and-avoid capabilities alone are not always sufficient, especially in these relatively high collision risk flight situations. See-and-avoid capabilities are not only limited by the physiological restrictions of human vision but also in some cases by unmitigated visual restrictions of the cockpit environment, such as when a faster low-wing aircraft descends and "lands" on a slower high-wing aircraft while both are on final approach to a runway. ${ }^{8}$ In such situations it would be useful to have an onboard capability that could augment pilots' see-and-avoid abilities with both alerts and also traffic avoidance guidance, preferably at lower altitudes and/or regardless of the intruder aircraft's equipage. One way to provide this capability for pilots of manned aircraft may be to leverage research and development efforts applied toward Detect and Avoid (DAA) capability for unmanned aircraft systems (UAS).

The National Aeronautics and Space Administration (NASA) has begun a DANTi (DAA iN The Cockpit) research effort that includes concept development, prototype implementation, integration and flight testing of NASA-developed DAA technology in manned aircraft cockpits to serve as a safety augmentation to pilots' see-and-avoid capabilities, rather than a replacement of see-and-avoid capabilities as in DAA for UAS. To the greatest extent possible the DANTi effort will leverage the current base of UAS DAA research and development work and, ideally, DANTi development and testing results will inform further the UAS community's DAA efforts, such as in lower-altitude operations near airports that are not fully covered by the present draft DAA standards.

This paper describes the initial development of a DANTi concept of use and prototype, and results from initial flight tests conducted with this first prototype. The next section of the paper briefly describes DAA systems and "well clear volumes," followed by sections that describe the DANTi concept of use and system options, respectively. Subsequent sections describe the initial DANTi prototype and initial flight test results, followed by a summary and conclusion section.

\section{DAA Systems and Well Clear Volumes}

NASA, in collaboration with the FAA, Department of Defense, industry participants and RTCA, Inc. (an aviation standards development organization), has been actively developing UAS DAA technology and standards recommendations for over five years. There is an extensive base of UAS DAA development work, described in the RTCA Special Committee 228 (SC-228) Phase I draft DAA Minimum Operational Performance Standards (MOPS) document ${ }^{11}$ (henceforth abbreviated to "DAA MOPS") and numerous NASA and additional publications (e.g., Refs. 12-18). The SC-228 UAS community's expectation going forward is that the FAA will reference the final published DAA MOPS as part of future DAA equipment standards, ultimately leading to additional rule changes as necessary and to operational approval for use of DAA systems on UAS flying in the National Airspace System. Phase I DAA systems are expected to have some operational restrictions placed upon them, and work on standards for Phase II DAA systems to remove some or all of those restrictions is now under way in the RTCA SC-228 community.

A UAS DAA system is expected to include one or more airborne sensors that provide aircraft traffic data over an air-ground data link to an algorithm that generates traffic alerts and maneuver guidance to a UAS pilot at a ground control station. DAA systems provide guidance to remain well clear of traffic by replacing a pilot's visual subjective judgment with a parametrically-defined "well clear volume" (WCV) around each nearby traffic aircraft or "intruder." That is, the DAA system's maneuver guidance is designed to keep the own aircraft ("ownship") outside of each intruder's WCV and thus "well clear."

The parametric shape and size of the DAA WCV has been the subject of extensive analysis and development within the UAS DAA community, and is described in detail in the DAA MOPS ${ }^{11}$ and other supporting documents (e.g., Refs. 12,13,16,17). The WCV is parametrically defined by distance-based horizontal (DTHR) and vertical (ZTHR) thresholds, and by a time-based "closure time" (TTHR) threshold. The WCV shape is complex and dependent on encounter geometry, but notionally it can be pictured as a cylinder centered on its respective intruder of radius DTHR and height ZTHR above and below the intruder, with an elongation in the direction of closure velocity between the intruder and ownship of a length proportional to TTHR and the closure rate. A mathematical description of the WCV is beyond the scope of this paper but compatibility with TCAS II is one of the rationales for its shape and size, such that aircraft that remain clear of each other's respective WCV will minimize TCAS RA occurrences at en-route altitudes if either aircraft is TCAS II equipped. Operationally, aircraft that maintain lateral or vertical separation greater than DTHR or ZTHR, respectively, throughout an encounter are considered well clear of each other. For converging encounters where both lateral and vertical separation will be lost, the TTHR threshold roughly

American Institute of Aeronautics and Astronautics 
approximates time to closest point of approach and thus a minimum "longitudinal well clear separation" that increases with higher closure rate encounters.

The WCV defined in the DAA MOPS is relatively large for several reasons. First, for UAS there is no see-andavoid mitigation so the collision-avoidance safety case for DAA is relatively more stringent than it might be if DAA were used for merely augmenting see-and-avoid traffic operations in manned aircraft as in DANTi. Second, the Phase I DAA MOPS is focused primarily on en-route operations away from the vicinity of airports and the WCV is designed for en-route (versus terminal-area) TCAS-compatibility. TCAS II will generate an RA at larger distances from an intruder when en-route than when at lower altitudes associated with an airport environment ${ }^{4}$ where aircraft separations are typically smaller. The relatively large DAA MOPS WCV may cause excessive false alerts when operating near airports, e.g., during approach, landing, takeoff and pattern operations, and if so would be a significant factor in the use of DAA in manned aircraft. Smaller WCV parameter threshold values were evaluated during the initial DANTi flight tests and results are reported later in this paper.

The reference maneuver guidance algorithm in the DAA MOPS is the NASA-developed DAIDALUS (Detect and Avoid Alerting Logic for Unmanned Systems, Ref. 11 Appendix G) and DAIDALUS is also the algorithm used in the DANTi effort. DAIDALUS was developed using formal methods that allow for formal proofs of algorithm correctness, and the implementing code for the algorithm has been extensively tested both in computer simulations against stressing case encounter geometries as well as in airspace simulation environments and multiple flight tests. ${ }^{14-}$ 17 DAIDALUS receives as input the WCV parameter thresholds, ownship and traffic position and velocity data, ownship performance data (e.g., assumed turn rate), wind information, and an Alerting Time value. If the ownship is predicted to penetrate an intruder's WCV within the Alerting Time then DAIDALUS provides alerts and maneuver guidance output in the form of ranges of acceptable headings, airspeeds, and vertical speeds to remain well clear of the intruder. If there are multiple intruder aircraft then DAIDALUS provides guidance ranges that will keep the ownship well clear of all such intruders.

\section{DANTi Concept of Use}

Since DANTi systems are intended to augment rather than replace pilots' see-and-avoid capabilities, their use by manned aircraft pilots will be somewhat different than the use of DAA by a UAS pilot. Pilots' see-and-avoid capabilities will continue to be the primary means of visual separation, with DANTi systems acting as a "second set of eyes" for conflicting aircraft that pilots may not have (yet) visually acquired. Like the ADS-B In based ATAS application (and UAS DAA), DANTi is designed to alert pilots of conflicting aircraft early enough that well clear separation can be maintained with normal flight maneuvers and before more aggressive collision avoidance maneuvers (e.g., TCAS RA) might be required. However, unlike the ATAS application (but like UAS DAA), DANTi systems will additionally provide suggested well-clear maneuver guidance along with the traffic alert. This comparison between ATAS and DANTi is best illustrated with an analogy to traffic advisory services provided to pilots by ATC. ATAS provides the aural equivalent of an ATC traffic call-out, for example, "traffic, 12 o'clock, opposite direction, 2 miles, same altitude" to alert the pilot and aid in visual acquisition and avoidance of the traffic, whereas DANTi additionally adds the visual equivalent of an ATC controller's heading suggestion, e.g. "suggest a right [or left] turn 20 degrees from present heading." ATC will provide pilots such maneuver suggestions on request and these suggestions are especially helpful to pilots when they have not visually acquired the traffic in question. Pilots understand that they are ultimately responsible for visual avoidance of all traffic and should scan for other traffic in the direction of the suggested avoidance turn before executing it, since ATC may not be aware of all nearby traffic or other flight constraints. This same understanding would apply when pilots use a DANTi system's suggested maneuver guidance to remain clear of aircraft they have not (yet) visually acquired: the pilot should visually clear the path in the direction of the avoidance maneuver for any aircraft not known to the DANTi system. The safety case for DANTi implementations to reliably detect all nearby conflicting traffic is thus arguably less stringent than for UAS DAA use where see-and-avoid capability is not present, and is arguably comparable to the current practice of pilots visually

clearing and then following ATC suggested avoidance maneuvers. This observation enables the consideration of simpler DANTi implementations with fewer than the full set of DAA sensors required for UAS applications.

\section{DANTi System Options}

DANTi implementations could take several forms. An implementation that closely tracked the DAA MOPS would consist of three onboard sensors (ADS-B In, radar, and TCAS-interrogation capability), a fusion/tracker capability to combine the sensor data, and a processor(s) to run the fusion/tracker and DAIDALUS algorithm to generate alerts and maneuver guidance for display on the pilots' panel-mounted flight instrumentation, e.g., the primary flight display and navigation display or multifunction display. The sensors and algorithms used in such an implementation would

American Institute of Aeronautics and Astronautics 
also require inputs from other onboard equipment such as an air-data and attitude-heading reference system and a radar altimeter. Such an implementation would maximize leverage of standards developed for UAS DAA and would be capable of detecting non-cooperative intruder aircraft, but it would be relatively expensive and because of its size, weight and power requirements, it would be more suited to larger, higher-end general aviation (GA) aircraft, especially those used by business aviation and charter operators that frequent busier non-towered airports.

Cost-sensitive operators such as small single-engine GA aircraft owners/pilots and even the rental aircraft community could potentially derive a safety benefit from simpler, lower-cost DANTi implementations. Such implementations might have only ADS-B In as a traffic sensor, and could be either installed systems that display DANTi maneuver guidance on the pilots' panel-mounted flight instrumentation or fully portable systems that display their guidance on a tablet or electronic flight bag (EFB) device. Fully portable systems could be especially costeffective and might reach a wide pilot audience including the renter-pilot community, trading off their lack of complete traffic detection (due to only a single sensor) with low cost and widespread use, and thus potentially maximizing their positive safety influence. These DANTi systems could be either standalone applications or integrated into other avionics applications and displays. It may even be advantageous to consider a combined ATAS and DANTi application as the two are not necessarily mutually exclusive; that is, the DANTi maneuver guidance might be presented to the pilot concurrently with any issued ATAS aural traffic alert. In all cases the intent of these future DANTi systems would be to capitalize on the new FAA policy ${ }^{19}$ announced 7 July 2016 intended to "reduce costs and streamline the introduction of Non-Required Safety Enhancing Equipment (NORSEE) into the general aviation fleet."

\section{Initial DANTi Prototype}

NASA has developed an initial prototype DANTi implementation that incorporates a single sensor (ADS-B In) and displays maneuver guidance on an EFB (for accuracy considerations this initial prototype does not compute or display maneuver guidance for TIS-B (Traffic Information Service - Broadcast) detected traffic, only directlyreceived air-to-air ADS-B transmissions). This DANTi implementation, shown in Fig. 1, was tested in a laboratory simulation environment and then installed and flown in NASA Langley Research Center's Cirrus SR22 research aircraft (Fig. 2) with NASA’s Cessna 206 aircraft (Fig. 3) flying various maneuvers near the SR22 as a traffic intruder.

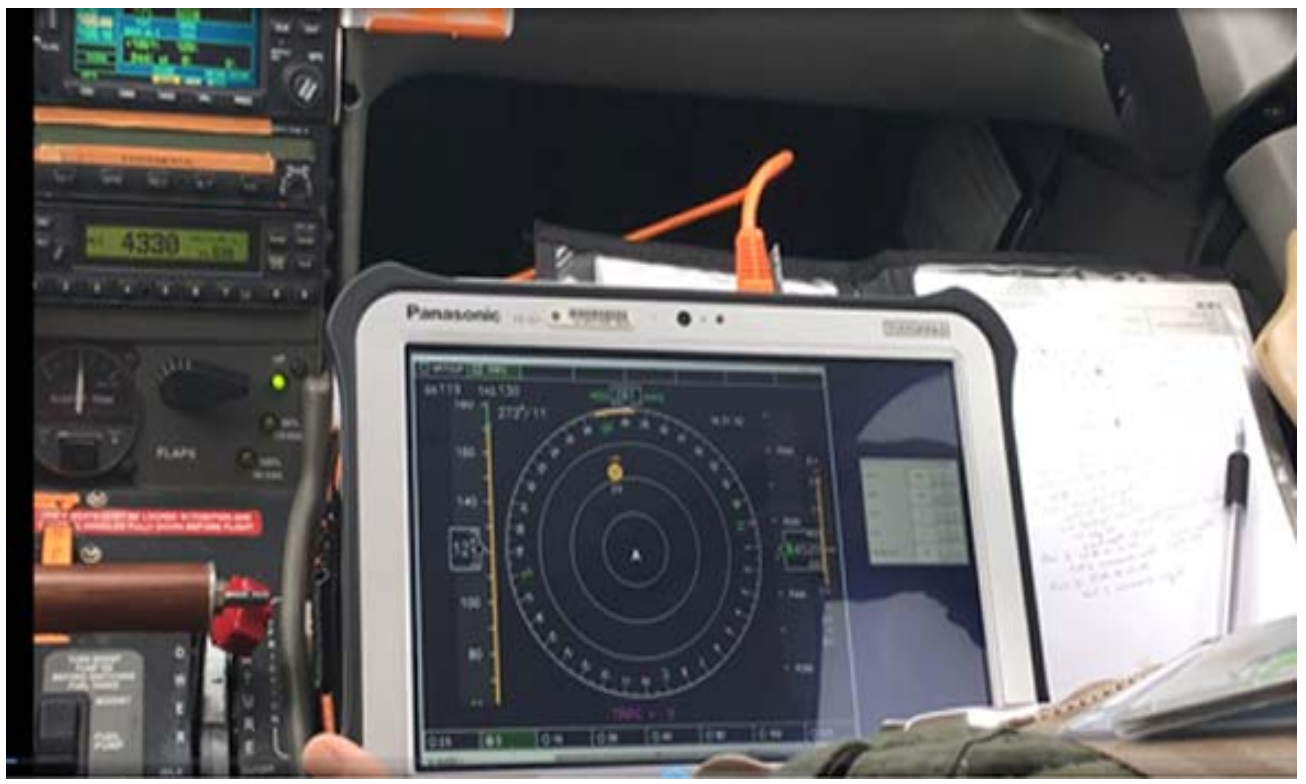

Figure 1. DANTi Prototype in Flight.

The DANTi implementation includes flight data input and processing software, the DAIDALUS maneuver guidance algorithm, and software to present DANTi display guidance to the pilot on the EFB. Ownship heading, airspeed and altitude information was obtained from onboard air data and heading reference research systems and supplied via an Ethernet connection (orange cable at top of EFB). For research purposes the implementation was developed to use a Linux-based operating system environment on the EFB but nothing in its design precludes modification for operation on other EFB or tablet devices. As shown in Fig. 1, the DANTi implementation presents the pilot with a magnetic-heading-up display of traffic information with an indicated airspeed tape to the left of this

American Institute of Aeronautics and Astronautics 
heading-up display and indicated altitude and vertical velocity tapes to the right. Groundspeed (GS) and true airspeed (TAS) are displayed near the top of the airspeed tape along with the wind magnetic direction and speed. The display range scale is selected at the bottom of the application and in the figure is set to five nautical miles (NM); the halfscale range ring on the traffic display (2.5 NM in this case) is also labeled to indicate the selected scale. The small gray rectangle on the EFB to the right of the DANTi application is a graphical user interface into DANTi for research purposes that allows the WCV and Alerting Time parameters to be dynamically changed during flight tests, as described in subsequent sections of this paper.

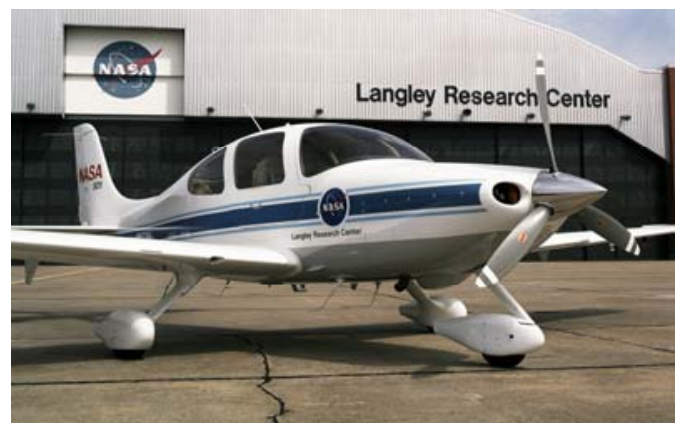

Figure 2. NASA Langley Cirrus SR22 aircraft (“Ownship”)

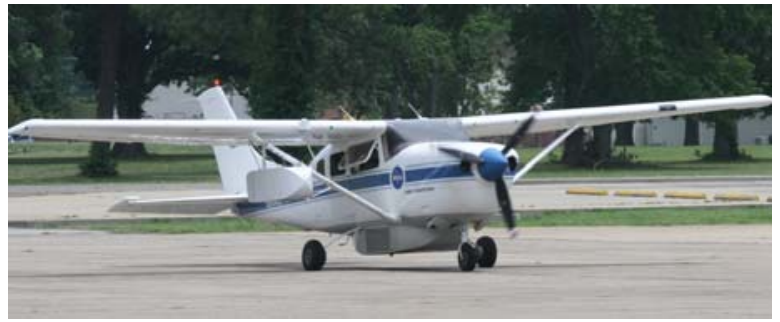

Figure 3. NASA Langley Cessna 206 (“Intruder”)

Figure 1 shows an opposite-direction flight encounter with the eastbound Cessna 206 intruder approximately 2.5 NM from the SR22 ownship and close enough to cause a loss of well clear separation (i.e., penetration of the WCV as defined by the selected parameters) within the selected Alerting Time, as indicated by the amber-circle intruder aircraft symbol (associated with a Caution level of alert) with an enclosed chevron pointing in the direction of its track, and the associated amber heading, airspeed and vertical velocity maneuver guidance bands. In all cases the tip of the chevron symbol indicates the actual aircraft location. The "corrective" guidance bands show that the defined well clear separation can be maintained by a small right heading change or a slightly larger left heading change to the respective edge of the amber heading guidance band, or by a sufficient descent or climb rate as indicated by the amber vertical velocity band. An airspeed change will not resolve this well-clear conflict (airspeed change guidance can be useful to resolve same-direction overtaking-aircraft conflicts). For operational DANTi systems it is envisioned that after the ownship maneuvers outside of either the displayed heading or vertical velocity guidance band ranges the intruder symbol would revert back to a white chevron, indicating that the conflict is resolved. However, for this research prototype the intruder symbol remains amber as long as even "preventive" maneuver guidance bands associated with the intruder are displayed (e.g., “don't turn left” preventive heading bands would remain just after a right deviation clear of this encounter). The intruder symbol reverts to a white chevron after all maneuver guidance bands associated with it are absent and all headings, airspeeds and vertical velocities can be selected without causing a well clear conflict with that intruder.

This initial DANTi prototype implementation employs a simpler alerting scheme than that described by the DAA MOPS for UAS, which employs a multi-level alerting scheme that includes, in addition to the Caution alert used by DANTi, an Advisory level alert and larger volume outside of the WCV as well as a Warning alert level for encounters more urgent than the Caution alert level. The rationale for DANTi's simpler alerting scheme is that manned aircraft pilots will be using DANTi as an augmentation of their see-and-avoid capabilities rather than a replacement, so a more comprehensive DANTi alerting scheme is arguably neither necessary nor desirable. The FAA recommends that in visual conditions pilots should spend approximately 70 percent of their "visual scan time" looking outside for traffic with the remaining 30 percent devoted to cockpit scans and other duties, ${ }^{20,21}$ so an overly complex alerting scheme and display may actually detract from a pilot's see-and-avoid responsibilities. Additional human factors studies in this area may be useful. 


\section{DANTi Initial Flight Tests}

One of the primary objectives of the two flight tests described in this section of the paper, beyond demonstrating an initial DANTi proof-of-concept, was make initial observations regarding the applicability of the DAA WCV configuration values established in the DAA MOPS.

Table 1. DANTi Parameter Threshold Sets

\begin{tabular}{|c|c|c|c|c|}
\hline & \multicolumn{4}{|c|}{ Threshold Set } \\
\hline DAIDALUS Parameter & 1 & 2 & 3 & 4 \\
\hline DTHR (feet) & 4000 & 1200 & 1200 & 1200 \\
\hline ZTHR (feet)* & (450) & $(300)$ & (300) & (300) \\
\hline TTHR (seconds) & 35 & 15 & 0 & 0 \\
\hline Alerting Time (seconds) & 40 & 40 & 40 & 20 \\
\hline
\end{tabular}

Four sets of threshold values were evaluated during these flights, shown as Sets 1 through 4 in Table 1 . Threshold Set 1 contains the DAA MOPS values: horizontal threshold (DTHR) of 4000 feet, vertical threshold (ZTHR) of 1200 feet, "closure time" threshold (TTHR) of 35 seconds, and Alerting Time of 40 seconds. Threshold sets 2 through 4 represent progressively smaller WCV and/or shorter Alerting Times. The DTHR and TTHR values in Set 2 correspond to the smallest TCAS II protected volume at which a TCAS RA may still be issued at low altitudes (so-called TCAS "Sensitivity Level 3"), specifically at altitudes between 1000 and 2350 feet above ground level such as when operating near airports. Set 3 further reduces the TTHR value to zero, producing a cylindrical WCV of radius DTHR and height of ZTHR above and below the aircraft, and Set 4 uses this same cylindrical WCV but with Alerting Time reduced from 40 to 20 seconds. DANTi was tested and evaluated in flight using each of these sets in December 2016, both in structured en-route encounter geometries as well as in a series of specific traffic pattern encounter geometries.

\section{A. First Flight Test}

The first DANTi research flight test was conducted at NASA Langley Research Center's local airfield (Langley Air Force Base, KLFI) on December 15, 2016, after two previous instrument check flights in November. This first research flight was conducted using only the DANTi-equipped SR22 aircraft conducting traffic pattern operations to KLFI's Runway 26, and a stationary ADS-B Out transmitter mounted on top of NASA's research hangar simulating a traffic aircraft, similar to the scenario of a helicopter hovering near the hangar.

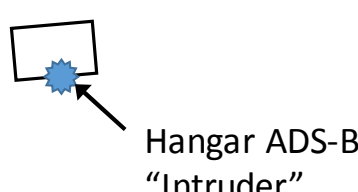

"Intruder"

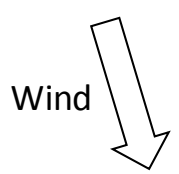

Mag. North

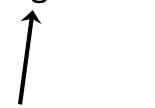

SR22

Ownship

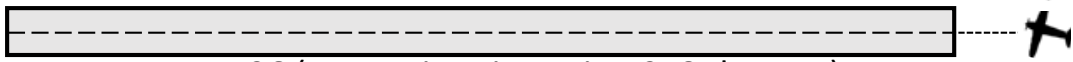

Runway 26 (magnetic orientation 258 degrees)

Length 10,000 feet

Figure 4. Langley (KLFI) Runway 26 approach. 
As shown in Fig. 4, Runway 26 is 10,000 feet long with a magnetic orientation of 258 degrees, and NASA's research hangar, with the intruder signal, is approximately 2800 feet (slightly less than $0.5 \mathrm{NM}$ ) to the right (North) of the runway near its distant end. There was a strong northwest wind during the test flight resulting in a significant right wind correction heading angle when the SR22's track was aligned on the Runway 26 centerline during final approach. Four low approaches were flown to Runway 26, one for each of the four DANTi threshold value sets in Table 1.

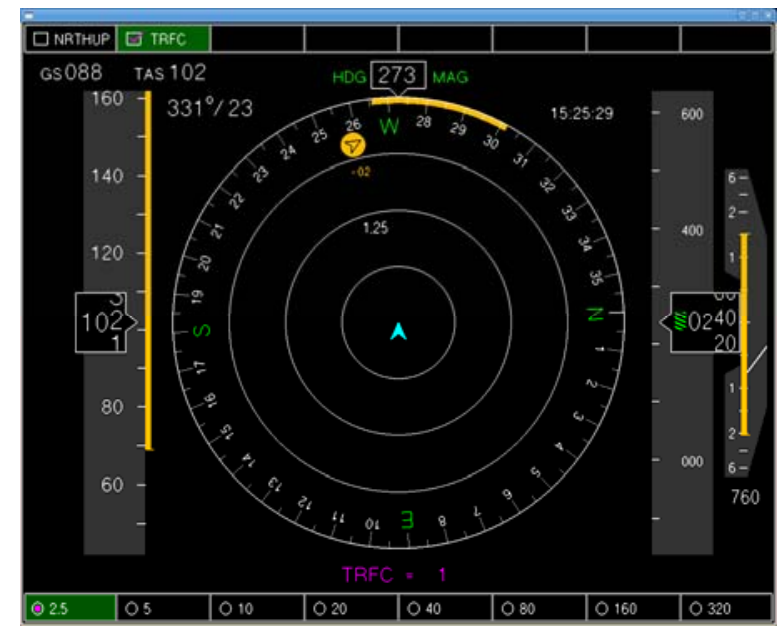

Figure 5. DANTi Alert and Guidance: Runway 26, Set 1.

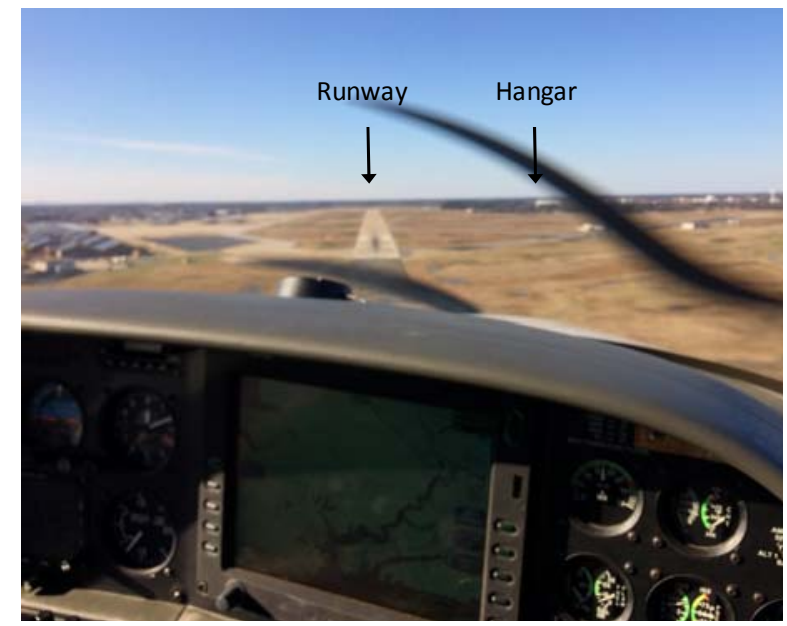

Figure 6. Out-the-Window View: Runway 26, Set 1.

Figure 5 shows a DANTi display image and Fig. 6 shows a photograph of the corresponding out-the-window view while on final approach to Runway 26 using Set 1 (the DAA MOPS threshold set). The DANTi image in Fig. 5 shows the display immediately after presentation of its traffic alert and maneuver guidance; the out-the-window view shown in Fig. 6 is annotated with arrows pointing out the runway and the hangar "traffic intruder" locations. Due to the 15degree right wind correction angle and the approximately 2 NM distance the "intruder" actually appears to the left of the aircraft's centerline even though the intruder is to the right of the aircraft's track along the runway's extended centerline.

Several observations can be made about the Set 1 flight encounter pictured in Figures 5 and 6:

1) First, the out-the-window sight picture of traffic and its corresponding depiction on a heading-up traffic display in the presence of a strong crosswind is significantly different from what would be shown on a trackup traffic display, and a heading-up display is arguably more useful in visually acquiring displayed traffic.

2) Second, due to Set 1's DTHR value of 4000 feet (which is larger than the hangar's runway offset of approximately 2800 feet), DANTi presents an unnecessary traffic alert and maneuver guidance, indicating that at least an 8 degree left turn is required to remain clear (which would cause the ownship to deviate left of the runway centerline).

3) Third, even if the DTHR value was made smaller to account for sufficient lateral separation with the intruder, it can still be argued that the alert is likely occurring too early; that is, while the ownship is still on short final to a 2-mile runway when the intruder is near the distant end of the runway. To illustrate this argument, consider if Runway 26 consisted only of its first 3000 feet (a reasonable and not-uncommon runway length used by small GA aircraft) and the helicopter was then hovering near its extended centerline but more than a mile past its distant end. In this case while the helicopter traffic would again be of interest it would not prevent a safe landing, and yet the resulting early alert and corrective maneuver guidance would indicate otherwise to the pilot. Delaying this type of early false alert requires smaller values for either the Alerting Time and/or TTHR values.

Sets 2 through 4 have progressively smaller Alerting Time and/or TTHR parameter values, and each have DTHR values of 1200 feet, so none caused corrective guidance during approaches to Runway 26. In each case, preventive guidance (i.e. “don't turn too far right”) was generated at progressively further distances down Runway 26 during the respective low approaches; note that if the "intruder" had been closer than 1200 feet to the runway centerline then in each case the guidance would have been corrective to remain well clear. Figure 7 shows the preventive guidance for Set 3, just after it was presented approximately 5000 feet before abeam the "intruder." Similar preventive guidance 
was presented for Sets 2 and 4, at approximately 6500 and 3500 feet before abeam the "intruder," respectively. In these cases, it appears that the DTHR value of 1200 feet is more appropriate than 4000 feet for avoiding false alerts in pattern operations when sufficient lateral separation with an intruder is present, and that the smaller Alerting Time and/or TTHR values in Sets 2 through 4 are progressively less likely to generate false alerts when sufficient longitudinal separation is present. However, in the longitudinal separation case it becomes increasingly unclear where lack of a false alert becomes a late or missed alert, and to some extent this relies on the pilot's subjective judgment.

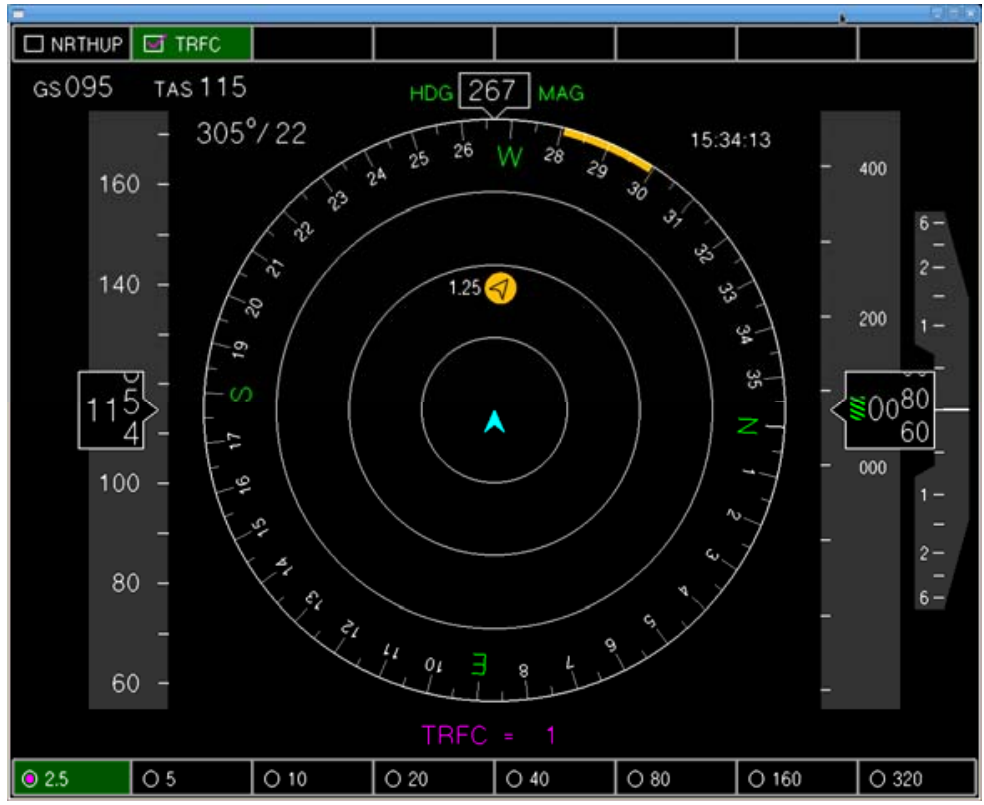

Figure 7. DANTi Alert and Guidance: Runway 26, Set 3.

\section{B. Second Flight Test}

The second DANTi flight test was conducted at and above Wakefield Municipal Airport (KAKQ), which is a rural, non-towered airport with very light GA traffic, located $31 \mathrm{NM}$ west of KLFI. Flight time for this test was approximately 2 hours, including transit time to and from KLFI, and used NASA's Cirrus SR22 aircraft as the DANTi ownship and NASA's ADS-B Out-equipped Cessna 206 (C206) as its intruder for flight maneuvers. Approximately the first half of the research flight time was devoted to simulated collision encounters at altitude above KAKQ and the remainder of the flight time was devoted to airport traffic pattern maneuvers between the two aircraft at KAKQ with no other aircraft in the vicinity of the airport. The simulated collision encounters were performed with a 500-foot vertical offset (SR22 and C206 level at 4500 and 5000 feet, respectively) for flight safety and assured separation, and the DANTi ZTHR parameter was set to 1000 feet (i.e. an artificially "tall” WCV) to assure generation of DANTi alerts and guidance when insufficient horizontal separation was predicted. Scripted opposite-direction and 90-degree converging encounters to a common point were flown with several of the four threshold sets from Table 1, and with or without following DANTi maneuver guidance, to allow for post-hoc quantitative analyses of DANTi maneuver guidance.

Three traffic pattern maneuvers were evaluated during the flights at KAKQ, with each maneuver flown four times using each of the four DANTi threshold sets (total of 12 traffic pattern maneuvers flown). These three maneuvers are diagrammed in Figures 8 through 10, respectively. The first maneuver, shown in Fig. 8, is most analogous to the "simulated hovering helicopter" encounter of the first flight test in regards to timeliness of alerts. In this maneuver,the C206 intruder lined up for takeoff on the runway in use and held in position while the SR22 turned onto an extended final approach to landing (a low approach was actually executed). The SR22 pilot confirmed visual sighting of the C206 holding at the beginning of the runway and continued inbound on final approach at 90 knots until the SR22 pilot judged that minimum safe longitudinal spacing existed with the C206 aircraft, at which point the SR22 pilot instructed the C206 pilot to commence takeoff. The researcher monitoring the DANTi display noted when its alert and corrective guidance was presented relative to when the SR22 pilot called for C206 takeoff. 


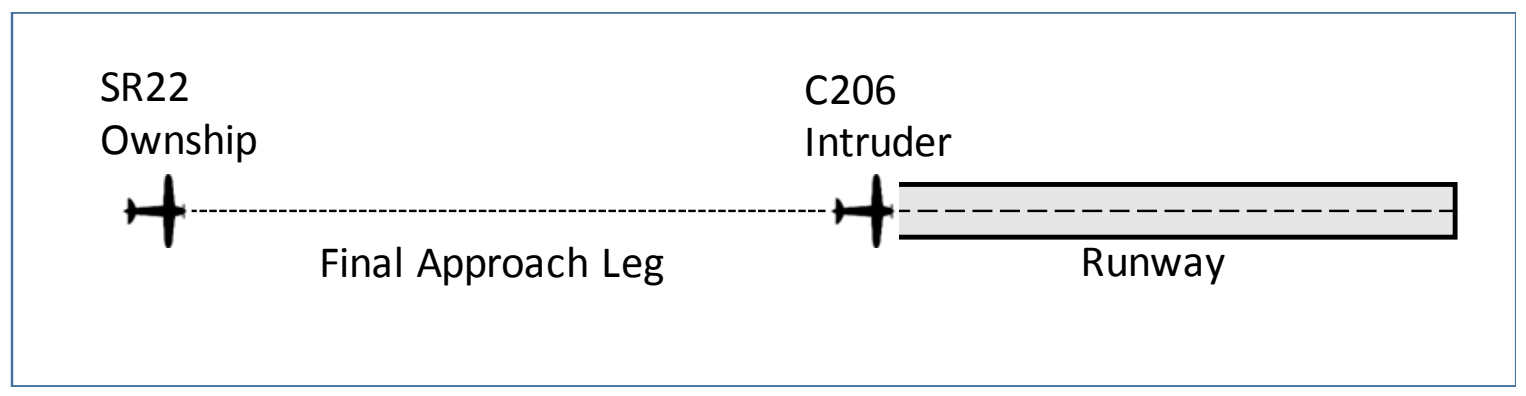

\section{Figure 8. Ownship on Final Approach, Intruder Holding on Runway.}

In the case of Set 1 the DANTi alert and corrective guidance occurred approximately 20 seconds before the SR22 pilot called for takeoff, while approximately 2 NM from the runway threshold and C206. Set 2 alerted at basically the same time that the SR22 pilot called for takeoff, at approximately 1.5 NM from the runway threshold, resulting in a "spot on" remark from the pilot regarding the alert timing. For Set 3 the pilot noted when he would call for takeoff but then continued for a short while further before making the actual call, resulting in a DANTi alert about 12 seconds after desired by the SR22 pilot. DANTi did not alert with Set 4 before the SR22 pilot called for takeoff and no attempt was made to determine how much time would have subsequently elapsed before a DANTi alert would have occurred. Collectively these results for the maneuver in Fig. 8 agree with the assessment from the first "simulated helicopter" flight test that the threshold values in Set 1 cause alerts to occur too early. In addition, the Set 2 values generated DANTi alerts and guidance for this maneuver that coincided with the subjective assessment of the NASA pilot, and Sets 3 and 4 generated late alerts with respect to the same pilot's subjective assessment.

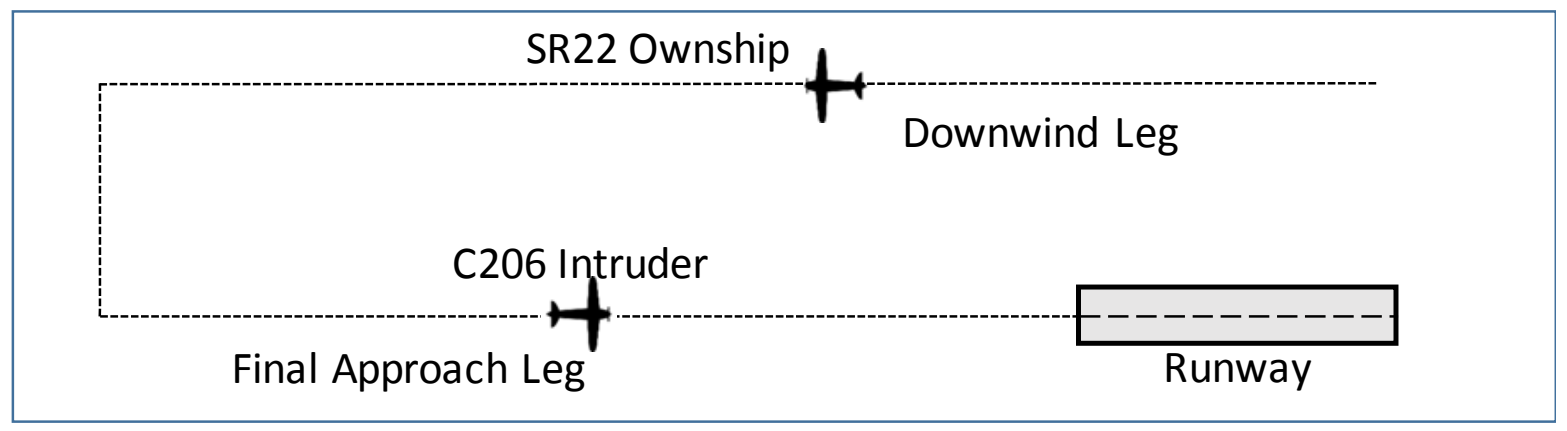

Figure 9. Ownship on Downwind Leg, Intruder on Final Approach.

Figure 9 shows the second traffic pattern maneuver, in which the SR22 on an extended downwind leg flies past the opposite-direction C206 intruder on extended final approach leg while both aircraft are at a pattern altitude of 1000 feet. The SR22 pilot was asked to maintain tight but safe lateral spacing of the downwind leg relative to final approach, consistent with tight spacing that might typically be used by small single-engine GA aircraft pilots. Set 1 generated a corrective guidance alert for this fly-by maneuver while sets 2 through 4 did not; this result is consistent with the first flight test and indicates that a DTHR value of 4000 feet is likely to produce false alerts during traffic pattern operations while a 1200 -foot value does not.

Figure 10 shows the third traffic pattern maneuver in which both aircraft are on the downwind leg but the SR22 is behind the C206 and overtaking it with 50 knots closing speed (SR22 and C206 flying at 130 and 80 knots, respectively). The maneuver began with the SR22 pilot verifying visual contact with the C206 while approximately 2 NM behind it, and then continuing the overtake maneuver until the SR22 pilot declared that, in his judgment, some action should be taken (e.g., slow down or maneuver clear) to maintain safe spacing. Set 1 values caused DANTi to provide an alert and corrective guidance approximately 20 seconds before the pilot's declaration, at a distance of approximately 1.5 NM behind the C206. Set 2 resulted in an alert about 10 seconds after the pilot would have preferred,

American Institute of Aeronautics and Astronautics 
approximately 1 NM in trail of the C206, with a pilot remark that the spacing was "a little uncomfortable" but still safe. The Set 3 and 4 overtake maneuvers were terminated before DANTi presented a corrective alert, which would have occurred with even smaller in-trail spacing than with Set 2.

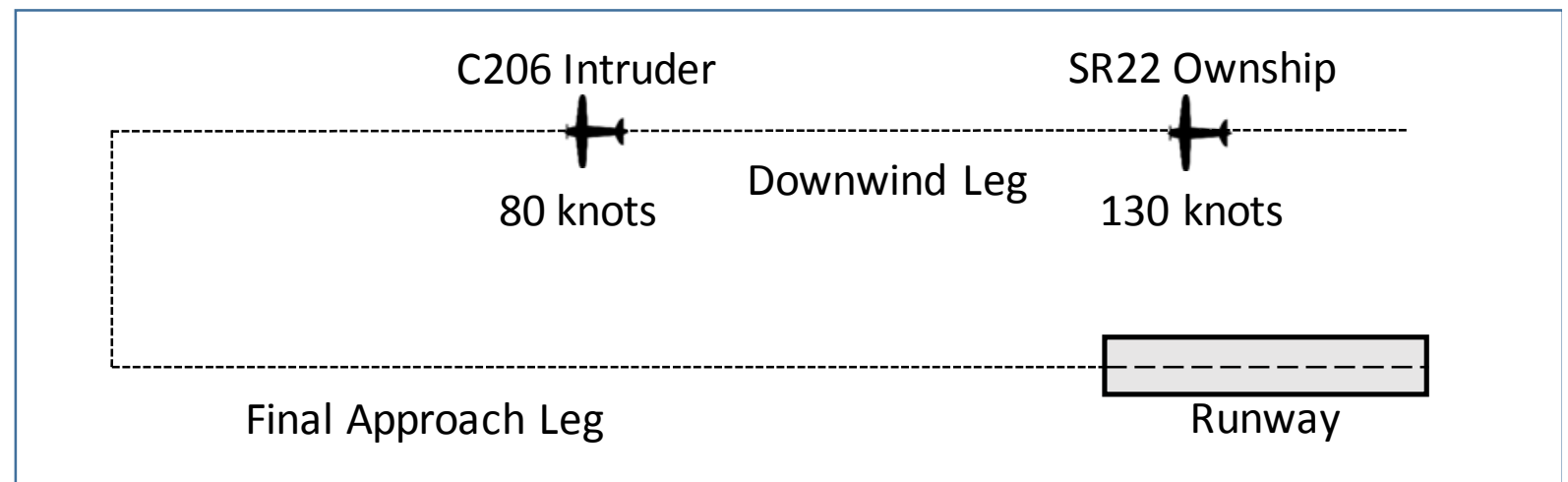

Figure 10. Ownship Overtaking Intruder on Downwind Leg.

Many caveats are associated with the observations made in the two brief, initial DANTi flight tests described in this paper, including the facts that only a limited number of threshold value sets and pattern maneuvers were tested, and that the subjective assessments of false or missed alerts are relative to the in-flight judgment of only a single NASA research pilot.

These caveats notwithstanding, results from these first-look flight tests indicate that the TTHR and DTHR threshold values specified in the Phase I DAA MOPS (Set 1) may be too large for use in traffic pattern operations and result in unnecessary false alerts, while the smaller TTHR and DTHR values used in Set 2 minimize false alerts while still providing timely alerts and guidance for traffic encounters. Sets 3 and 4 represent progressively smaller WCV sizes and Alerting Times, and based on the results of these flight tests may be too small to generate timely alerts and maneuver guidance.

One of the lessons learned from this initial set of flight tests is the importance of wind vector information for the correct presentation of maneuver guidance to the pilot. In its absence, maneuver guidance would be presented relative to ground track irrespective of aircraft heading, which may cause the traffic display and guidance to be confusing and/or misleading.

\section{Summary and Conclusion}

Mid-air collision risk continues to be a concern for manned aircraft operations, especially near busy non-towered airports, and the use of DAA technologies and draft standards developed for UAS, either alone or in combination with other collision avoidance technologies such as ATAS or TCAS II, may be useful in mitigating this collision risk for manned aircraft. The paper has described the various forms that such a DAA system could take for use in a manned aircraft, and the tradeoffs between low cost and complete traffic detection that might drive the design of these various forms. NASA's DANTi research effort has been described, including the initial development of a DANTi concept of use and prototype, and the initial flight tests conducted with this prototype. The prototype used a single ADS-B traffic sensor and ownship position, track, heading and air data information, along with the NASA-developed DAIDALUS reference algorithm for the UAS DAA MOPS, to display traffic alerts and maneuver guidance to manned aircraft pilots on a portable EFB. Initial flight tests with the prototype demonstrated a successful DANTi proof-of-concept, but also demonstrated that the traffic separation threshold values specified in the Phase I DAA MOPS may generate excessive false alerts during traffic pattern operations. Several sets of smaller separation threshold values were also tested in flight, one of which yielded more timely alerts for the maneuvers tested.

The DANTi prototype and flight test results described in this paper are only the initial steps necessary for a longerterm DANTi research effort. Future work could include flight tests of more separation threshold sets, and evaluation of more traffic pattern maneuvers and potential collision geometry encounters with additional pilots. Flight tests with additional sensors such as UAS traffic radars would also be useful, including evaluations and tests of new lower-cost radar sensors with more limited detection ranges. The initial flight tests described in this paper have demonstrated the value of heading-up traffic displays and the availability of wind information for computation of maneuver guidance, but these features require not only ownship heading information but also airspeed data; these data may be available

American Institute of Aeronautics and Astronautics 
from newer production GA avionics but more portable installations may have only heading data, and further study is required to determine the efficacy of such systems. Finally, additional human factors studies would be useful to further inform the optimal DANTi alerting structure and pilot interface.

It is anticipated that the results described in this paper, in combination with future DANTi simulation and flight test results, will inform development of future RTCA standards recommendations and guidelines for future operational approval of DANTi implementations.

\section{Acknowledgments}

This work was sponsored and funded by the National Aeronautics and Space Administration. Any software endeavor or flight test is a team effort and the authors gratefully acknowledge the exceptional team members that made this effort possible, including but certainly not limited to Richard Grube, Charles Howell, Brian Hutchinson, Jerry Karwac, Kevin Shelton, Greg Slover and Taylor Thorson.

\section{References}

${ }^{1}$ Federal Aviation Administration, “Traffic Alert and Collision Avoidance System (TCAS) Airborne Equipment, TCAS I,” Technical Standard Order Number TSO-C118a, October 2014.

${ }^{2}$ RTCA, Inc., "Minimum Operational Performance Standards for an Active Traffic Alert and Collision Avoidance System I (Active TCAS I),” Document DO-197A, Special Committee No. 147, RTCA September 1994, Washington, DC.

${ }^{3}$ Federal Aviation Administration, “Traffic Alert and Collision Avoidance System (TCAS) Airborne Equipment, TCAS II With Hybrid Surveillance,” Technical Standard Order Number TSO-C119e, June 2016.

${ }^{4}$ RTCA, Inc., "Minimum Operational Performance Standards (MOPS) for Traffic Alert and Collision Avoidance System II (TCAS II) Hybrid Surveillance,” Document DO-300A, Special Committee No. 147, RTCA March 2013, Washington, DC.

${ }^{5}$ Federal Aviation Administration, “Avionics Supporting Automatic Dependent Surveillance - Broadcast (ADS-B) Aircraft Surveillance Applications (ASA),” Technical Standard Order Number TSO-C195b, September 2014.

${ }^{6}$ RTCA, Inc., "Minimum Operational Performance Standards (MOPS) for Aircraft Surveillance Applications (ASA) System,” Document DO-317B, Special Committee No. 186, RTCA June 2014, Washington, DC.

${ }^{7}$ Kunzi, F., and Hansmon, R. J., "Mid-Air Collision Risk and Areas of High Benefit for Traffic Alerting," AIAA Paper 20116894, 11th AIAA Aviation Technology, Integration and Operations (ATIO) Conference, September 2011.

${ }^{8}$ Federal Aviation Administration, "How to Avoid a Mid Air Collision," Document No. P-8740-51, URL: https://www.faasafety.gov/gslac/ALC/libview_normal.aspx?id=6851 [cited 31 March 2017].

${ }^{9}$ Title 14 United States Code of Federal Regulations Chapter I Subchapter F Part 91 Section 91.113, "Right-of-Way Rules: Except Water Operations,” Doc. No. 18334, 54 FR 34294, Aug. 18, 1989, as amended by Amdt. 91-282, 69 FR 44880, July 27, 2004.

${ }^{10}$ Federal Aviation Administration, “Airworthiness Approval for ADS-B In Systems and Applications,” Advisory Circular Number 20-172B, May 2015.

${ }^{11}$ RTCA, Inc., "Minimum Operational Performance Standards (MOPS) for Detect and Avoid (DAA) Systems," RTCA Paper Number 261-15/PMC-1400, Special Committee No. 228, RTCA December 2016, Submitted for Publication.

${ }^{12}$ Consiglio, M., Chamberlain, J., Munoz, C., and Hoffler, K., “Concept of integration for UAS operations in the NAS," in Proceedings of the 28th International Congress of the Aeronautical Sciences, (Brisbane, Australia), September 2012.

${ }^{13}$ Munoz, C., Narkawicz, A., Chamberlain, J., Consiglio, M., and Upchurch, J., “A Family of Well-Clear Boundary Models for the Integration of UAS in the NAS," AIAA Paper 2014-2412, 14th AIAA Aviation Technology, Integration, and Operations (ATIO) Conference, June 2014.

${ }^{14}$ Upchurch, J., Muñoz, C., Narkawicz, A., Chamberlain, J., and Consiglio, M., “Analysis of Well-Clear Boundary Models for the Integration of UAS in the NAS,” NASA TM-2014-218280, June 2014.

${ }^{15}$ Consiglio, M., et al., "Human-In-The-Loop Experimental Research for Detect and Avoid,” Proceedings of the 34th Digital Avionics Systems Conference (DASC 2015), Prague, Czech Republic, 2015.

${ }^{16}$ Munoz, C., Narkawicz, A., and Chamberlain, J., “A TCAS-II resolution advisory detection algorithm,” AIAA Paper 20134622, AIAA Guidance Navigation, and Control Conference and Exhibit, August 2013.

${ }^{17}$ Munoz, C., Narkawicz, A., Hagen, G., Upchurch, J., Dutle, A., and Consiglio, M., "DAIDALUS: Detect and Avoid Alerting Logic for Unmanned Systems,” Proceedings of the 34th Digital Avionics Systems Conference (DASC 2015), Prague, Czech Republic, September 2015.

${ }^{18}$ Chamberlain, J., Consiglio, M., Comstock, J. R., Ghatas, R., and Muñoz, C., “NASA Controller Acceptability Study 1 (CAS1) Experiment Description and Initial Observations,” NASA TM-2015-218763, May 2015.

${ }^{19}$ Federal Aviation Administration, “Approval of Non-Required Safety Enhancing Equipment (NORSEE),” Policy Statement Number PS-AIR-21.8-1602, March 2016.

${ }^{20}$ Federal Aviation Administration, "Scanning for Other Aircraft," Aeronautical Information Manual, Section 8-1-6(c), December 2015.

${ }^{21}$ Federal Aviation Administration, "Pilots’ Role in Collision Avoidance,” Advisory Circular Number 90-48D Change 1, September 2016.

American Institute of Aeronautics and Astronautics 